\title{
Resistant kawasaki disease in an infant causing giant coronary aneurysms with thrombosis
}

\author{
Ayad Anass $^{1 *}$, Hasbaoui Brahim ${ }^{1}$, Sghir Salah ${ }^{1}$, Yajouri Abdelhakim ${ }^{1}$, Abilkassem Rachid ${ }^{1}$, Hassani Amale $^{1}$, Agadr Aomar $^{1}$ and Faid Soumia $^{2}$ \\ ${ }^{1}$ Department of Pediatrics, Military Hospital of Rabat, Morocco \\ ${ }^{2}$ Department of Cardiology, Military Hospital of Rabat, Morocco
}

\begin{abstract}
Giant coronary artery aneurysms that occur in 0.5 to $1 \%$ of patients with Kawasaki disease can be fatal if associated with thrombosis. Some patients may show persistent inflammation and fever despite treatment with repeated doses of intravenous immunoglobulin (IVIG), steroids, and aspirin.

This report describes an infant boy with resistant Kawasaki disease who presented with extensive coronary artery involvement and coronary thrombosis. His inflammation was not controlled with multiple doses of IVIG, parenteral and oral steroids, or high-dose aspirin, and he finally needed infliximab, a monoclonal antibody against tumor necrosis factor alpha.
\end{abstract}

\section{Case details}

A 13 month-old boy male infant admitted for fever, generalized polymorphous exanthema, hyperemic conjunctivae, and diarrhea. The onset of the disease was approximately 2 weeks before the admission with a generalized polymorphous exanthema for which he received antihistaminic treatment, but after 7 days he associated fever, hyperemic conjunctivae, and diarrhea being admitted in the regional hospital. The fever persisted despite the antibiotic and symptomatic treatment, and therefore he was transferred in our hospital.

The clinical exam at the time of admission revealed influenced general status, generalized polymorphous exanthema, hyperemic pharynx, bilateral conjunctivitis, and approximately five diarrheic stools per day. The laboratory tests revealed leukocytosis $(27,050 /$ $\mathrm{mL}$ ), with neutrophilia $(19,900 / \mathrm{mL}$ ), anemia ( $\mathrm{Hb} 6.9 \mathrm{~g} / \mathrm{dL}$, Htc 19.7\%, MEV $68.6 \mathrm{fL}, \mathrm{MEH} 24 \mathrm{pg}$ ), thrombocytosis $(1,111,000 / \mathrm{mL})$, elevated inflammatory biomarkers (CRP $62.01 \mathrm{mg} / \mathrm{L}$, ESR $36 \mathrm{~mm} / \mathrm{h}$ ), hypoalbuminemia $(2.8 \mathrm{~g} / \mathrm{dL})$, and mild hyponatremia $(\mathrm{Na} 135$ $\mathrm{mmol} / \mathrm{L}$ ). The cultures performed from stools, blood and urine were all negative. The abdominal ultrasound and the chest X-ray did not reveal any pathological elements. The serological test for Toxoplasmosis, Rubella, Herpes virus, Cytomegalovirus, Epstein-Barr virus, and viral hepatitis were negative. With a diagnosis of Kawasaki's disease, the patient received intravenous immunoglobulin (IVIG) without any clinical response.

His echocardiogram 2 days later showed diffuse dilation of both coronary arteries, with pericardial effusion. His remittent fever with continued inflammatory activity warranted a second dose of IVIG followed by pulse methyl prednisolone and infliximab (a monoclonal antibody against tumor necrosis alpha) infusion. The boy experienced a recurrence of fever with leukocytosis, thrombocytosis, and elevation of inflammatory markers. An electrocardiogram showed diffuse ST elevation, suggesting pericarditis. A repeat echocardiogram showed persistent diffuse dilation of the entire right coronary artery measuring $11 \mathrm{~mm}$ from origin to crux and also extending into the posterior descending coronary branch. The proximal and mid left anterior descending coronary artery (LAD) measured $9 \mathrm{~mm}$, together with ectasia of the left main coronary artery as well as the proximal and mid left circumflex artery (Figures 1 and 2).

Due to persistent ongoing inflammation, the boy received a third dose of IVIG together with high-dose aspirin, oral prednisolone, and

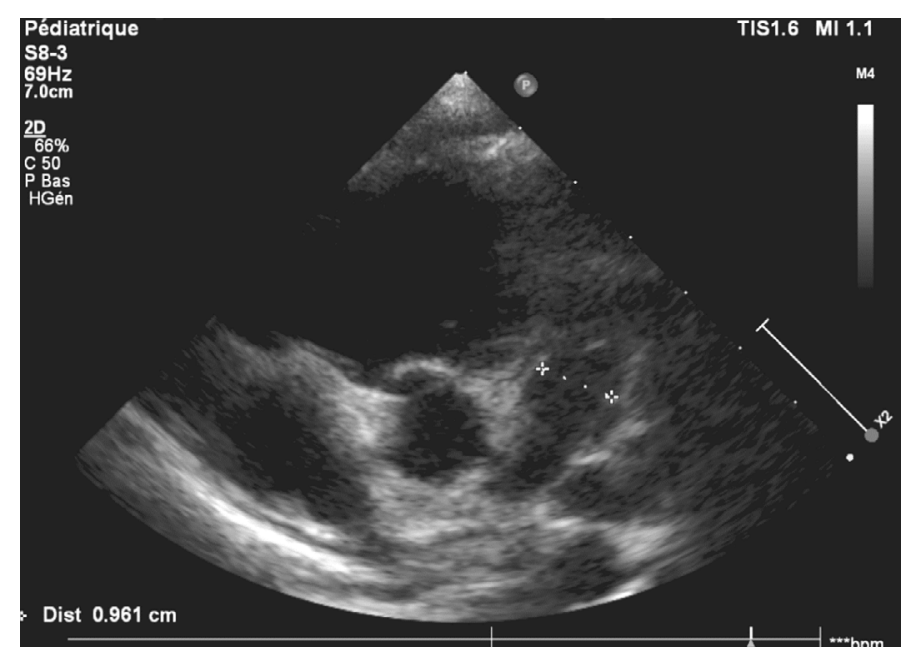

Figure 1. Parasternal short-axis view of the dilated left coronary artery. The proximal left anterior descending coronary artery (LAD) is aneurysmally dilated, and the left circumflex artery $(\mathrm{Cx})$ also is diffusely dilated

${ }^{\star}$ Correspondence to: Ayad Anass, Department of Pediatrics, Military Hospital of Rabat, Morocco, Tel: +212661315979, E-mail: drayadanass@gmail.com

Key words: resistant Kawasaki disease, intravenous immunoglobulin, coronary thrombus, aneurysm, infliximab

Received: September 30, 2020; Accepted: October 12, 2020; Published: October 19,2020 


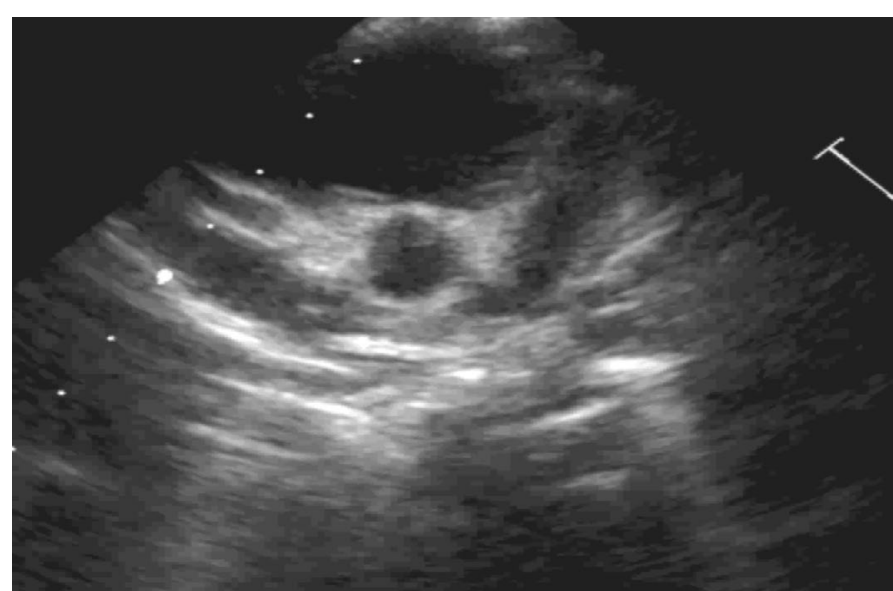

Figure 2. Parasternal short-axis view at the level of the aortic root demonstrating a proximal right coronary artery (RCA) aneurysmally dilated

warfarin. His platelet count rose to $10019109 / 1$ on day 28 of his illness. He had an erythrocyte sedimentation rate of $90 \mathrm{~mm}$ in first hour, and his C-reactive protein level was $90 \mathrm{mg} / \mathrm{l}$. A loose mobile thrombus measuring $1293 \mathrm{~mm}$ in the distal RAD responded to $24 \mathrm{~h}$ of abciximab infusion, with a marked reduction in thrombus size together with some reduction in the size of the right coronary artery aneurysm. The boy subsequently remained afebrile.

He was discharged on full-dose aspirin together with clopidogrel, alternate-day prednisolone and warfarin to maintain an international normalized ratio (INR) of 2 to 3.2 weeks after discharge. An echocardiogram showed a very small thrombus, with persistent dilation of the coronary arteries. At the 9-month follow-up visit, the boy was receiving warfarin and low-dose aspirin and showed normal growth and development. At this writing, he is scheduled for coronary imaging after a year

\section{Discussion}

Extremes of age represents, associated with male gender, prolonged fever and a delay in diagnosis or treatment an important risk factor for the development of giant coronary aneurysms, with thrombus noted in $0.5 \%$ to $1 \%$ of patients with Kawasaki's disease [1]. A coronary aneurysm is diagnosed when the internal lumen diameter of the coronary arteries is found to be $>3 \mathrm{~mm}$ in children younger than 5 years or $>4 \mathrm{~mm}$ in children older than 5 years on two-dimensional echocardiography.

The American Heart Association has classified coronary aneurysms on the basis of their sizes as small $(<5 \mathrm{~mm})$, medium $(5-8 \mathrm{~mm})$, and giant $(>8 \mathrm{~mm})$ [2]. Giant coronary aneurysms are less likely to regress, and they have the highest association with progression to stenosis and myocardial infarction [3].

Half of the patients develop at least transient coronary artery dilation and $1 \%$ of patients develop giant aneurysms. Half of these aneurysms resolve within 1-2 years of the acute phase. Because of myointimal proliferation, these patients are prone to develop thrombosis and stenosis of the affected segments, thus warranting serial follow-up with ultrasound. With the evolution of newer computed tomography scanner technology, the radiation exposure from an ECG-gated cardiac CTA has declined to under $1 \mathrm{mSv}$. In addition, CTA allows visualization of mid and distal segments, which are common sites for aneurysm formation and are poorly seen on the ultrasound. CTA is also helpful in assessing the degree of stenosis, presence of calcifications, thrombosis, and findings of embolic myocardial infarction and serves as a tool for non-invasive follow-up imaging in selected patients [4].

The current American Heart Association guideline favors low-dose aspirin for aneurysms up to $6 \mathrm{~mm}$ for a period of 8 weeks or until the aneurysms regress in size. Long-term antiplatelet treatment in patients with aneurysm more than $6 \mathrm{~mm}$ is recommended to avoid catastrophic sequel of myocardial infarction and death [5]. Intravenous immune globulin (IVIG), the cornerstone of therapy, can reduce the risk of coronary-artery aneurysms to $4 \%$. However, among the therapies tested so far that have been found to be effective against IVIG-resistant Kawasaki's disease, the best choice remains a subject of controversy [6]. Kawasaki's disease is characterized by a cytokine storm, with elevated levels of tumor necrosis factor (TNF) $\alpha$, interleukin-6, granulocyte colony-stimulating factor, interleukin- $1 \beta$, and interleukin-17.1 [1].

However, the boy's fever recurred despite infliximab, thereby necessitating prolonged oral steroid use. Extensive coronary artery aneurysms with thrombosis warranted the use of abciximab, another murine monoclonal antibody against platelet glycoprotein $2 \mathrm{~b} / 3 \mathrm{a}$ receptor [7]. Abciximab may promote vascular remodelling and could lead to regression of coronary aneurysms. If coronary thrombosis leads to myocardial infarction, thrombolytics may have a role [8]. Coronary angiography is needed to document regression of coronary artery lesions that may occur in $50 \%$ to $67 \%$ of aneurysms, often within the first 1 or 2 years [9]. Regression of aneurysms is more likely in young infants and in cases with smaller aneurysms, a fusiform aneurysm morphology, and a location in a distal coronary segment.

\section{Conclusion}

The present report illustrates a rare case of Kawasaki disease with a delayed presentation at 13 months of age, giant coronary aneurysms, and an accompanying coronary arterial thrombus that was detected by twodimensional echocardiography. Where Kawasaki disease is refractory to intravenous immunoglobulin therapy, methylprednisolone and infliximab may be used to reduce acute vascular inflammation.

\section{Conflict of interest}

The authors have no conflicts of interest to disclose.

\section{References}

1. Koren G, Lavi S, Rose V, Rowe R (1986) Kawasaki disease: review of risk factors for coronary aneurysms. J Pediatr 108: 388-392. [Crossref]

2. Gerding R (2011) Kawasaki disease: a review. J Pediatr Health Care 25: 379-387. [Crossref]

3. Horigome H, Sekijima T, Miyamoto T (1997) Successful thrombolysis with intracoronary administration of tissue plasminogen activator in an infant with Kawasaki disease. Heart 78: 517-518. [Crossref]

4. Ghoshhajra BB, Lee AM, Engel LC, Celeng C, Kalra MK, et al. (2014) Radiation dose reduction in pediatric cardiac computed tomography: experience from a tertiary medical center. Pediatr Cardiol 35: 171-179. [Crossref]

5. Newburger JW, Takahashi M, Gerber MA, Gewitz MH, Tani LY, et al. (2005) Diagnosis, treatment, and long-term management of Kawasaki disease: a statement for health professionals from the Committee on Rheumatic Fever, Endocarditis, and Kawasaki Disease, Council on Cardiovascular Disease in the Young, American Heart Association. Pediatr 115:1118.

6. Mori M, Imagawa T, Yasui K, Kanaya A, Yokota S (2000) Predictors of coronary artery lesions after intravenous gammaglobulin treatment in Kawasaki disease. $J$ Pediatr 137:177-180. [Crossref]

7. Burns JC, Mason WH, Hauger SB, Janai H, Bastian JF, et al (2005) Infliximab treatment for refractory Kawasaki syndrome. J Pediatr 146: 662-667. [Crossref] 
Anass A (2021) Resistant kawasaki disease in an infant causing giant coronary aneurysms with thrombosis

8. Kato H, Sugimura T, Akagi T, Sato N, Hashino K, Maeno Y (1996) Long-term consequences of Kawasaki disease: a 10- to 21-year follow-up study of 594 patients. Circulation 94:1379-1385. [Crossref]
9. Williams RV, Wilke VM, Tani LY, Minich LL (2002) Does abciximab enhance regression of coronary aneurysms resulting from Kawasaki disease? Pediatr 109: E4.

Copyright: (C2021 Anass A. This is an open-access article distributed under the terms of the Creative Commons Attribution License, which permits unrestricted use, distribution, and reproduction in any medium, provided the original author and source are credited. 